Journal of Vibration and Acoustics 127 (2) 139-143 (2005)

\title{
WAVELET ANALYSIS OF STICK-SLIP SIGNALS IN OSCILLATORS WITH DRY-FRICTION CONTACT
}

\author{
Jin-Wei Liang \\ Department of Mechanical Engineering, MingChi Institute of Technology, \\ Taipei, Taiwan, 24306, R.O.C. \\ Brian F. Feeny \\ Department of Mechanical Engineering, Michigan State University, \\ East Lansing, Michigan 48824-1226, U. S. A.
}

\begin{abstract}
Wavelet transforms were compared between various simulated friction models and real stick-slip data. While simulations of several models produced stick-slip transition oscillations seen in the real data, the wavelet features of the compliant contact model with light damping best captured the characteristics of the experimental signal. The wavelet contours were also used to estimate the contact stiffness.
\end{abstract}

\section{INTRODUCTION}

We characterize stick-slip motion by examining wavelet-transform contour plots corresponding to stick-slip responses in an experiment and in simulations of three different friction models. We then evaluate whether the friction model can successfully describe observed transition-oscillation dynamics. Stick-slip displacement, velocity, acceleration and friction force measurements from an mass-spring experiment [1] are shown in Figure 1. Transition oscillations occurring at the start of the sticking phase can be observed in the friction force and acceleration measurements. Intermittent high- and low-frequency stick-slip response components are well suited for wavelet analysis. If the wavelet transform successfully draws out these intermittent features, it may make a good tool for evaluating friction models. This study compares wavelet features of three simulated stick-slip models with an experiment.

Choosing a proper friction model is not always straight forward, and may benefit from additional evaluation tools. Oscillators with rigid Coulomb friction contacts have abrupt jumps in the friction force, and hence the acceleration, at the stick-slip transitions [2-4]. More complicated friction models [5-12] bring out finer details of stick-slip transitions. The goal is to explore the time/frequency transition features associated with different friction models. These wavelet transition features can be used for analyzing the experimental stick-slip signals, and evaluating friction models.

The wavelet transform has been applied to previous friction studies. Liang and Feeny [13] used 
the wavelet transform to detect the existence of subsystem dynamics occurring during the transition of stick-slip friction process. Jalali et al. [14] used the wavelet transform to detect the presence of stick-slip phenomena in granular flows. Other friction-vibration studies include $[15,16]$.

(a)

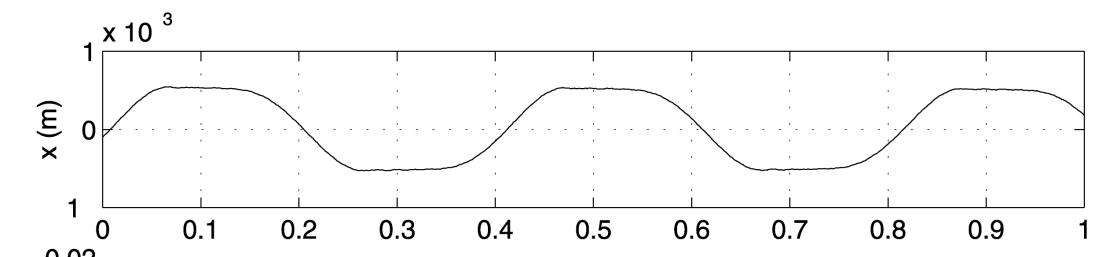

(b)

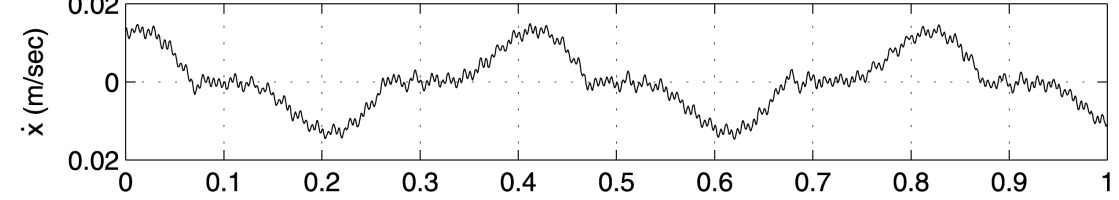

(c)

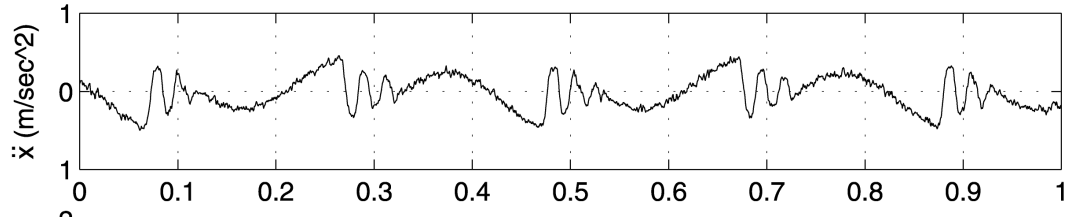

(d)

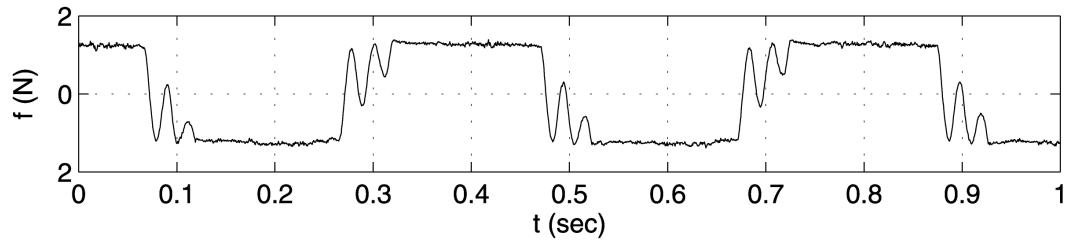

Figure 1: Experimental stick-slip signals (a) displacement, (b) velocity, (c) acceleration, and (d) friction force responses.

In this study, the wavelet transform is implemented using numerical-integration scheme [13, 17]. The mother wavelet is chosen as the Morlet wavelet while a dyadic grid was applied to discretize the scale-time plane. By numerically integrating the wavelet transform of acceleration signals, wavelet coefficients corresponding to different frequency scales and time positions can be obtained, and represented as wavelet contour plots. Thus, comparisons focusing on signatures registered in the wavelet-contour plots can be made.

\section{WAVELET TRANSFORM}

The wavelet transform is an inner product of a signal $x(t)$ and a particular set of functions $h(t)$, as

$$
W_{x}(a, b)=\int_{-\infty}^{\infty} x(t) h_{a, b}^{*}(t) d t
$$

where $h_{a, b}^{*}(t)$ represents the complex conjugate of $h(t)$. Equation (1) measures the "similarity" between the signal $x(t)$ and the basis functions 


$$
h_{a, b}(t)=\frac{1}{\sqrt{|a|}} h\left(\frac{t-b}{a}\right)
$$

called wavelets, in which $a, b \in \Re, a \neq 0$, and the constant $1 / \sqrt{|a|}$ is used for energy normalization (Önsay and Haddow [18], Staszewski [19]). The parameters $a$ and $b$ determine the dilation and translation of the mother wavelet which is chosen here as the Morlet wavelet (Morlet and Arens [20], Mallat [21], and Strang and Nguyen [22]) given by

$$
\begin{aligned}
& h(t)=\pi^{-1 / 4}\left(e^{-i \omega_{c} t}-e^{-\omega_{c}^{2} / 2}\right) e^{-t^{2} / 2} \\
& H(\omega)=\pi^{-1 / 4}\left[e^{-\left(\omega-\omega_{c}\right)^{2} / 2}-e^{-\omega_{c}^{2} / 2} e^{-\omega^{2} / 2}\right]
\end{aligned}
$$

where $\omega_{c}$ is the center frequency of the mother wavelet. $H(\omega)$ represents the Fourier transform of $h(t)$. The wavelet basis functions have no DC component, i.e. $H(\omega)$ evaluated at $\omega=0$ is zero. The second term in the bracket on the right-hand side of Equation (3) exists for the purpose of reconstructing (or inverting) the process. In practice, it can be neglected (Önsay and Haddow [18]). Therefore, it will not be included in our calculations.

The analyzing wavelet function, $h(t)(H(\omega))$, can be considered as a window function in both the time and frequency domains. Equations (3) and (4) state that the time window $h(t)$ is centered at $t=0$, whereas the frequency window $H(\omega)$ is centered $\omega=\omega_{c} . \quad$ When the translation and dilation actions are switched on, the time window will be centered at $t=b$ and the frequency window at $\omega=\omega_{c} / a$ as shown in Equation (2).

In order to implement the calculation of the wavelet transform, a sub lattice is constructed by discretizing the values of $a$ and $b$. Fixing the dilation and translation step size to $a_{o}$ and $b_{o}$, and defining $a=a_{o}^{m} ; b=n b_{o} a_{o}^{m}$, with $m, n \in Z$, results in

$$
h_{m, n}(t)=a_{o}^{-m / 2} h\left(a_{o}^{-m} t-n b_{o}\right)
$$

Based on Equation (5), the translation step $b$ depends on the dilation step $a$. This choice is natural, since long wavelets will then advance by large steps and shorts ones by small steps. On this discrete grid, the wavelet transform is thus

$$
W_{x}(m, n)=a_{o}^{-m / 2} \int_{-\infty}^{\infty} x(t) h^{*}\left(a_{o}^{-m} t-n b_{o}\right) d t .
$$

Of particular interest is the discretization on a dyadic grid which occurs for $a_{o}=2, b_{o}=1$ and is used in this study. To implement the calculation of wavelet coefficients, $W_{x}(m, n)$, the numerical integration scheme is adopted. This algorithm may not be efficient computationally, but the number of data points in this study is not huge. The same algorithm was used by Kishimoto et al. [17] 
successfully in studying a flexible beam vibration problem and by Liang and Feeny [13] for a transient friction vibration investigation. There are many other algorithms which can be found in the signal process literature (Mathworks [23], Newland [24]).

(a)

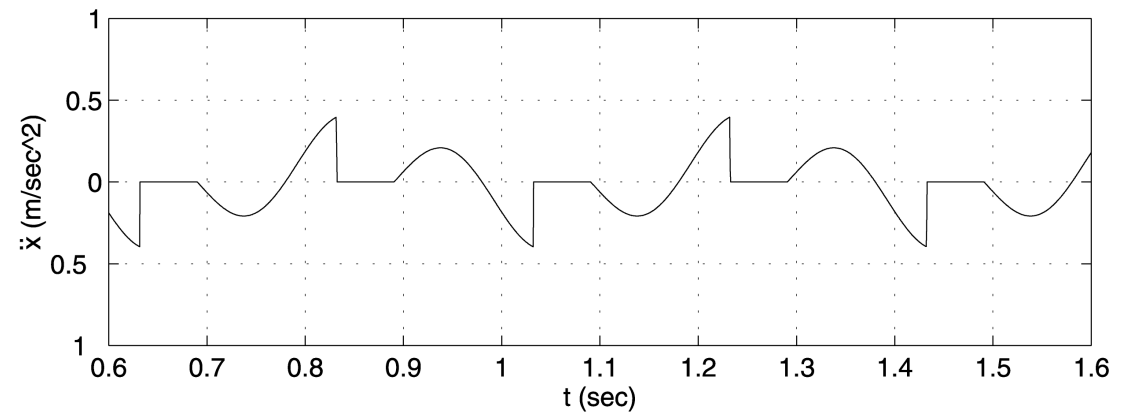

(b)

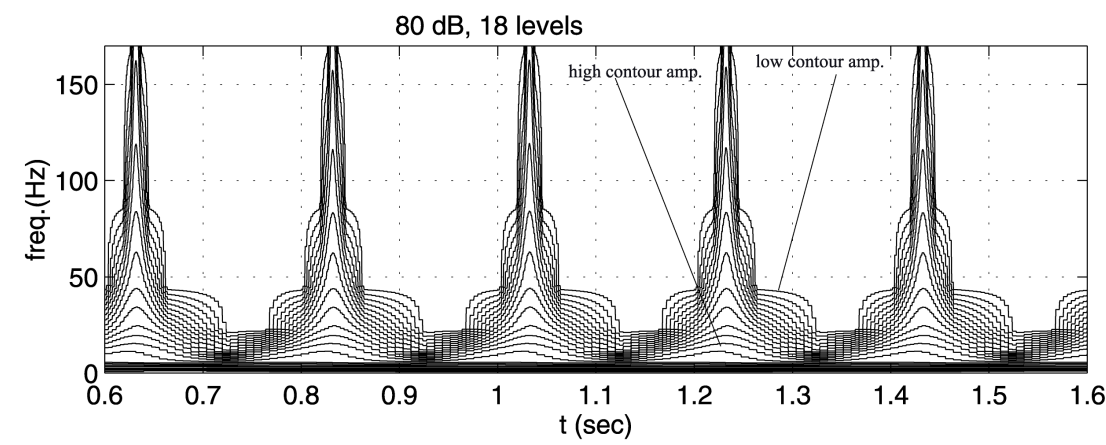

Figure 2: Wavelet analysis of the simulated stick-slip acceleration, Coulomb friction model: (a) time-domain response, and (b) wavelet contour plot.

We have tested the numerical computation scheme to the benchmarks signals such as sinusoids and impulses. It gives reasonable results [25]. In the next section, this method will be used to investigate both simulated and experimental acceleration signals that contain stick-slip motion.

\section{WAVELET TRANSFORM OF STICK-SLIP SIGNALS}

In this section, Coulomb, state-variable, and compliant-contact friction models are used to imitate the real stick-slip process shown in Figure 1. Wavelet contour plots are generated for the simulated stick-slip signals, and finally for the experimental stick-slip signal.

\subsection{Wavelet Analysis of the Coulomb Model Stick-Slip Acceleration Signal}

The harmonically forced Coulomb oscillator can be written as

$$
m \ddot{x}+k x+f_{k} f(\dot{x})=k Z_{e} \cos (\omega t)
$$

where $f(\dot{x})=-1, \dot{x}<0 ; f(\dot{x})=1, \dot{x}>0$; and $-1 \leq f(0) \leq 1$. Also, $f_{k}$ denotes the kinetic friction and $Z_{e}$ represents the amplitude of base excitation. The kinetic and static friction are modeled as equal. The main system parameters are: $m=2.42 \mathrm{~kg}, k=2,310 \mathrm{~N} / \mathrm{m}$, and $f_{k}=1.28 \mathrm{~N}$, whereas the excitation parameters include: $Z_{e}=0.0006 \mathrm{~m}$, and $\omega=2.5 \mathrm{~Hz}$.

The stick-slip acceleration signal and its wavelet transform are presented in Figure 2, in which a 
jump event takes place at each onset of sticking. Note that high-frequency information is contained in those acceleration jumps while the zero-value acceleration appearing during the rest of the sticking interval corresponds to a DC displacement behavior. The presence of both high- and low-frequency information in this time-domain signal makes the wavelet transform valuable. The wavelet contour plot presented in Figure 2(b) shows spikes at the jump events. The maximum wavelet coefficient repeats at the forcing frequency $(2.5 \mathrm{~Hz})$. There are 18 contour curves spanning $80 \mathrm{~dB}$ between the maximum wavelet coefficient and the threshold. In what follows, a constant span $(80 \mathrm{~dB})$ will be applied to different simulation cases and the experimental data for consistent comparison.

\subsection{Wavelet Analysis of the State-Variable Model Stick-Slip Signal}

Next, the stick-slip process of the state-variable friction oscillator is studied. In the state-variable friction model, there is an additional friction state which evolves dynamically about some backbone friction curve. Additional dynamics can be captured by this friction state. This study adopts a Coulomb law as the backbone friction characteristic, since experimental observations [1] showed little friction-velocity dependence. A similar choice was made by Dahl in his state-variable model [26]. An example [27] of an oscillator with a state-variable friction model is

$$
m \ddot{x}+k x(t)+\theta(t)=k Z_{e} \cos (\omega t)
$$

and

$$
\dot{\theta}(t)=-f_{k} \gamma(\theta(t)-f(\dot{x}))
$$

where $\theta(t)$ denotes the friction force as a state that tracks the backbone, steady-state friction characteristic $f(\dot{x})$. For the convenience of numerical integration, a smooth version of a Coulomb model, $\tanh (\alpha \dot{x})$, with a large value of $\alpha$, is implemented.

The system parameters used in the state-variable model simulation are $m=2.42 \mathrm{~kg}$, $k=2,310 \mathrm{~N} / \mathrm{m}, f_{k}=1.28 \mathrm{~N}, Z_{e}=0.0006 \mathrm{~m}, \omega=2.5 \mathrm{~Hz}, \alpha=3000$, and $\gamma=100$. The simulated stick-slip signal and its wavelet-transform contour plot are presented in Figure 3 . Unlike the Coulomb case, no abrupt jump event appears in the acceleration response. Rather, transition oscillation occurs during what would otherwise be the "sticking" interval. Thus, the state-variable chosen here brings forth a transition oscillation, which occurs during the transition from slip to stick. The transition oscillation is somewhat similar to that illustrated in Figure 1(c).

The wavelet transform contour plot depicts the transition oscillation shown in Figure 3(a) with less-pronounced spikes in comparison with those of the Coulomb case. Furthermore, two spikes rather than one appear whenever the mass sticks. These spikes point at two sharp "kinks" in the time-domain signal. In the Coulomb case, only one spike and one sharp kink show up. 
(a)
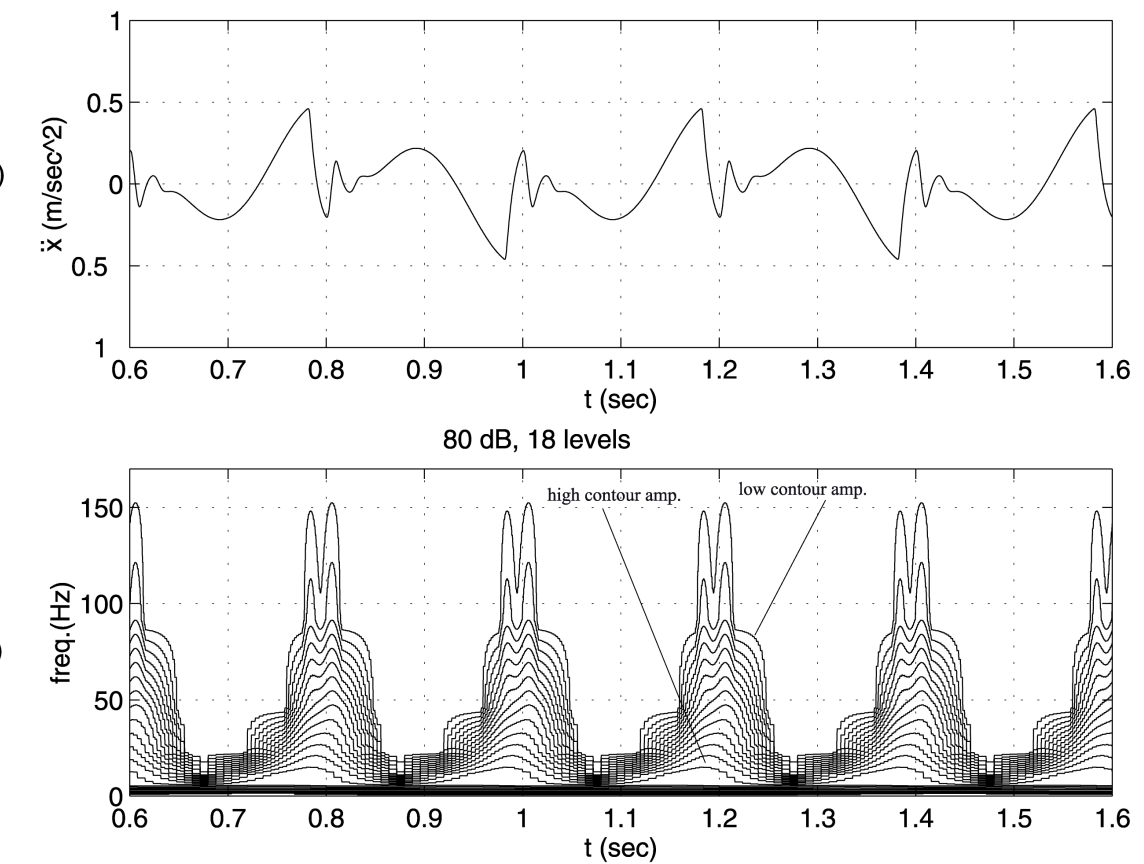

Figure 3: Wavelet analysis of the simulated stick-slip acceleration, state-variable friction model: (a) time-domain response, and (b) wavelet contour plot.

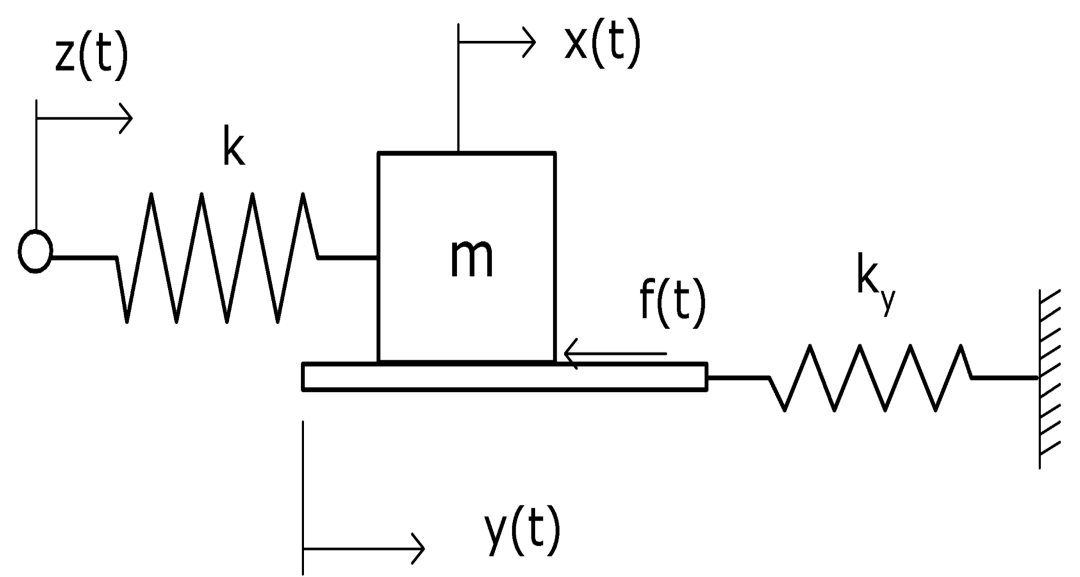

Figure 4: A schematic diagram showing the massless compliant contact model.

\subsection{Wavelet Analysis of the Compliant-Contact Model Stick-Slip Signal}

Finally, a compliant-contact friction model is investigated. A schematic diagram illustrating the forced Coulomb oscillator together with the idealized compliant-contact model with tangential stiffness is presented in Figure 4, in which $x(t)$ represents the displacement response of the sliding mass, $z(t)$ denotes the base excitation motion, $y(t)$ depicts the displacement response of the hypothetical contact surface, and $K_{y}$ represents the stiffness of the contact joint. Coulomb friction 
with equal static and kinetic friction is modeled at the surface.

The equation of motion is

$$
m \ddot{x}+k x+f=k Z_{e} \cos (\omega t)
$$

where $Z_{e}$ is the amplitude of a harmonic base motion, and $f$ represents the non-constant friction force occurring at the contact interface. The friction force $f$ can function in two different ways depending on the relative motion occurring at the contact interface. First, during the sliding interval the mass (Figure 4) moves relative to the contact surface, and the massless contact surface is assumed to be motionless. In that case, $y(t)= \pm Y_{m}= \pm f_{k} / K_{y}$, whereas $f(t)= \pm f_{k}$. There are no dynamics in $y$ during the sliding phase and the friction force takes constant values.

"Microsticking" starts when the relative velocity is zero, or $\dot{x}(t)=0$. During microsticking, both the sliding mass and the contact surface undergo the same motion such that $\dot{x}(t)=\dot{y}(t)$, until the static friction force can no longer sustain the stiffness force exerted by the compliant contact joint, i.e. $\left|K_{y} y(t)\right|>f_{k}$. The motion is called microsticking since when $K_{y}$ is large, both the sticking interval and the elastic displacement are small. Based on the above description, the model in the sliding phase is

$$
m \ddot{x}+k x+f_{k} \operatorname{sgn}(\dot{x})=k Z_{e} \cos (\omega t),
$$

and during the microsticking phase, the model becomes

$$
\begin{aligned}
& m \ddot{x}+k x+c \dot{y}+K_{y} y(t)=k Z_{e} \cos (\omega t) \\
& y(t)=Y_{m} \operatorname{sgn}\left(\dot{x}\left(t_{1}^{-}\right)\right)+x(t)-X_{s}
\end{aligned}
$$

where $Y_{m}$ (positive number) is the maximum deflection of the contact and $X_{s}$ (number with a sign) is the displacement of the mass before microsticking motion begins, $t_{1}$ represents the time instant at which the mass sticks. A damping factor is employed in the simulations of the sticking motion. The damping effect is only added to the contact model, so that $f(t)=K_{y} y(t)+c \dot{y}(t)$, and is neglected during sliding, under the assumption that sliding contact dynamics are fast due to the large value of $K_{y}$ and small contact mass. The system parameters for simulation are $m=2.42 \mathrm{~kg}$, $k=2,310 \mathrm{~N} / \mathrm{m}, f_{k}=1.28 \mathrm{~N}, Z_{e}=0.0006 \mathrm{~m}, \omega=2.5 \mathrm{~Hz}, K_{y}=209900 \mathrm{~N} / \mathrm{m}, c=80 \mathrm{~N}-\mathrm{sec} / \mathrm{m}$ for the heavier damping case, and $c=25 \mathrm{~N}-\mathrm{sec} / \mathrm{m}$ for the lighter damping case.

Figure 5(a) demonstrates the simulated stick-slip acceleration signal $(c=80 \mathrm{~N}-\mathrm{sec} / \mathrm{m})$ in which the transition oscillation is very close to a damped sinusoid. The wavelet transform contour plot corresponding to this simulated stick-slip signal illustrates two striking features. First, a local 
maximum of wavelet coefficients emerges at the transition oscillation, seen as a circular contour centered at about $45 \mathrm{~Hz}$. The small circular contour indicates a local maximum because the global maximum is actually a $2.5 \mathrm{~Hz}$ plateau existing along the entire time axis, corresponding to the low frequency forced response. Secondly, only one major spike appears in every transition from sliding to sticking rather than two spikes as the state-variable case does in Figure 3(b). These features imply that the transition oscillation in this case resembles a single damped harmonic function, which oscillates at $45 \mathrm{~Hz}$. Thus, the wavelet contour plot of this case is different from those of the state-variable and the Coulomb cases in which no local maximum exists during the stick-slip transition.

We can obtain quantitative modeling information from the $45 \mathrm{~Hz}$ microstick oscillation. During microsticking, the oscillator behaves like a mass attached with high contact stiffness in parallel to the softer spring attachment. The total stiffness (dominated by the contact stiffness) can be estimated as $\hat{K}=(2 \pi 45)^{2} m=193,500 \mathrm{~N} / \mathrm{m}$, which is a reasonable estimate of the total stiffness used in the simulation. This estimate is limited by the resolution of our contour plots.

If we slightly decrease viscous-damping effect in the compliant contact model, the micro-slip phenomenon will emerge in the transition oscillations [1]. To illustrate the phenomenon, the acceleration signal and its associated wavelet-transform plot of this lighter damping case $(c=25 \mathrm{~N}-\mathrm{sec} / \mathrm{m})$ are presented in Figures 6(a) and 6(b). Some observations can be made from Figures 6(a) and 6(b). First, similar to the heavier damping case, there are local maximum structures occurring in Figure 6(b). This local maximum contour describes the nearly sinusoidal feature of the transition oscillation presented in Figure 6(a). Secondly, several grouped spikes occur during each transition interval illustrating the occurrences of the microscale stick-slip events. The grouped-spike structure has not been observed in the Coulomb, state-variable, or even the compliant-contact model with heavier damping. The so-called double (two-scale) stick-slip event takes place during the macroscopic sticking interval, which occurs because the friction force, during the transition, temporarily reaches its maximum values twice before it finally stays at that value for a macroscopic sliding phase. When the friction force reaches its maximum value during the macroscopic sticking interval, the sliding mass will break free for a short time and micro-slip event occurs [1]. Corresponding to the transition oscillation, the wavelet contour plot again shows a local maximum at approximately $45 \mathrm{~Hz}$, indicating the frequency of the transition oscillation, and leading to the same contact stiffness estimation as above. 
(a)

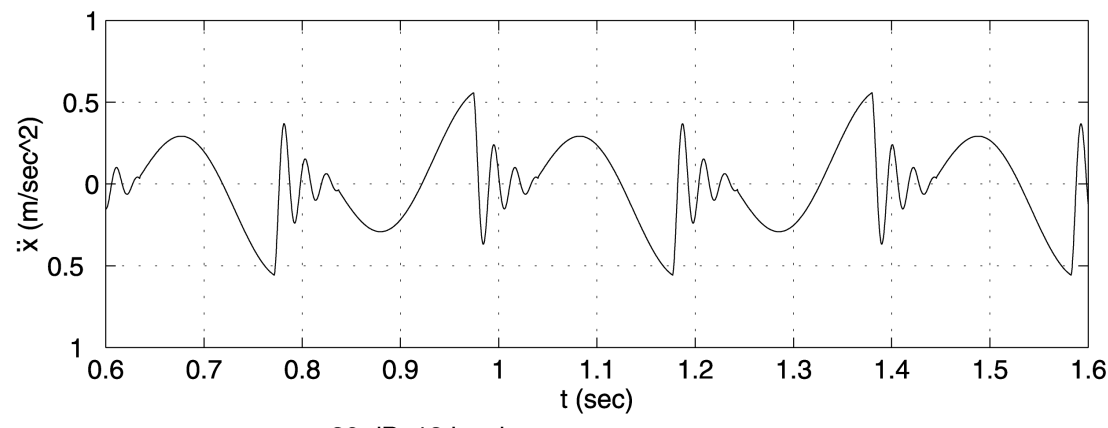

$80 \mathrm{~dB}, 18$ levels

(b)

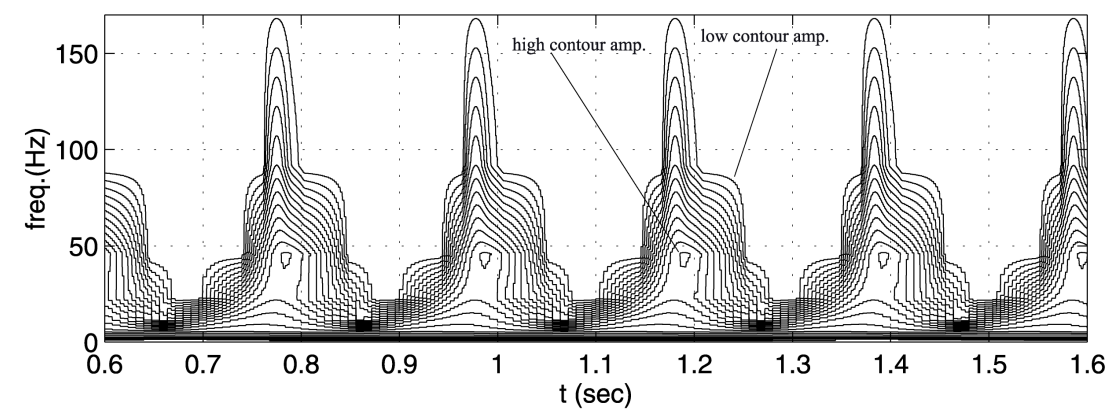

Figure 5: Wavelet analysis of the simulated stick-slip acceleration, tangential contact model with heavier viscous damping ( $\xi=0.0558)$ : (a) time-domain response, and (b) wavelet contour plot.

(a)
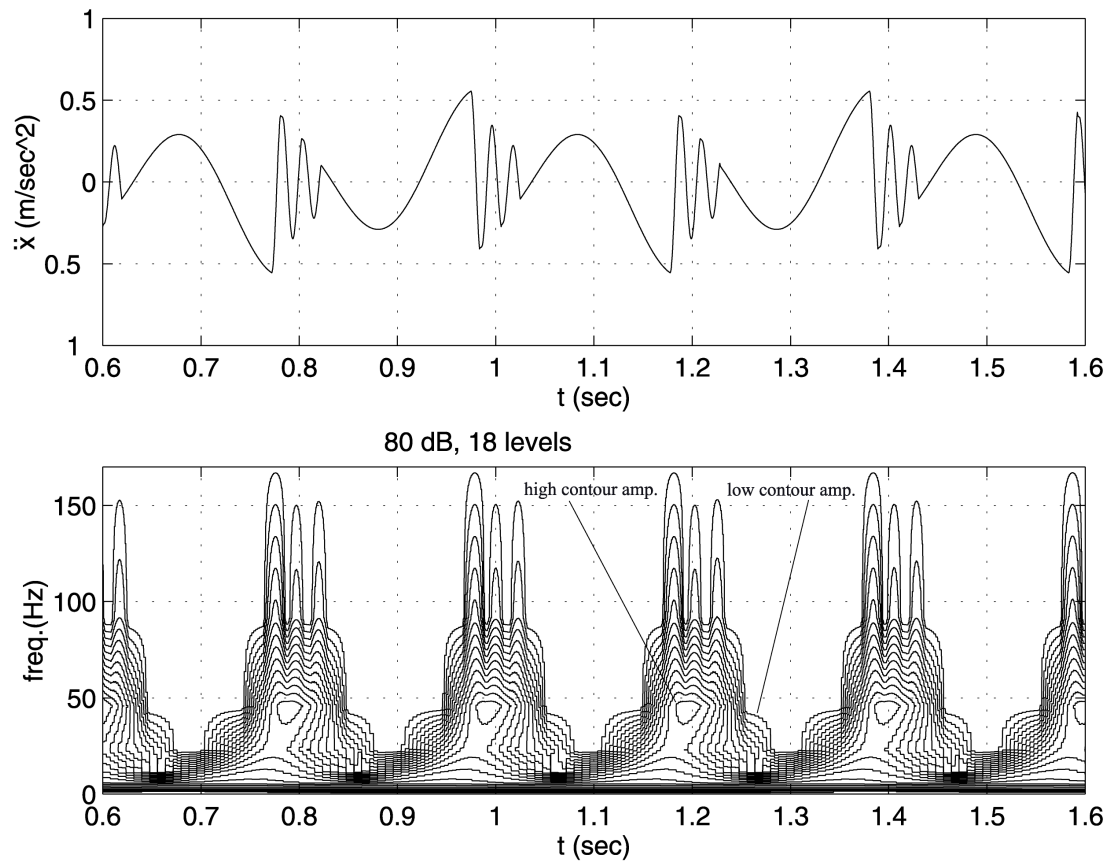

Figure 6: Wavelet analysis of the simulated stick-slip acceleration, tangential contact mode with lighter viscous damping $(\xi=0.0174)$ : (a) time-domain response, and (b) wavelet contour plot. 
(a)

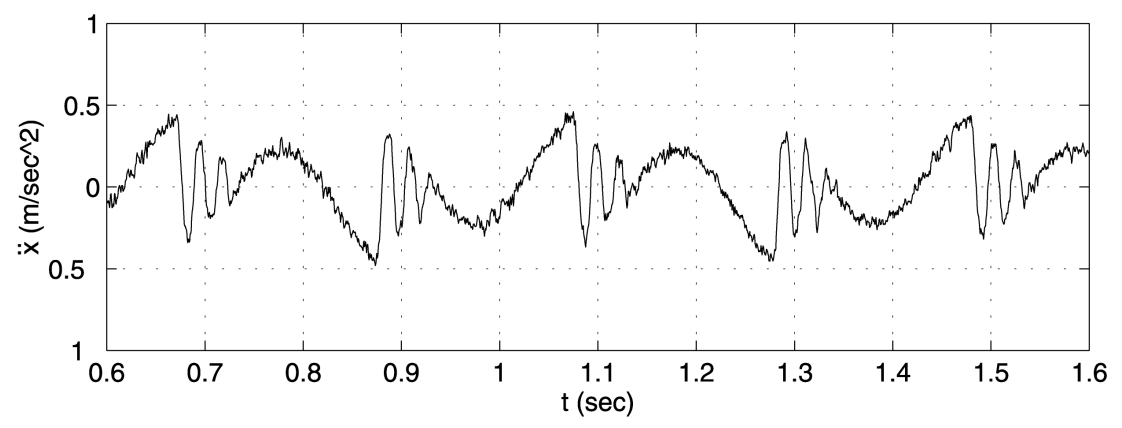

(b)

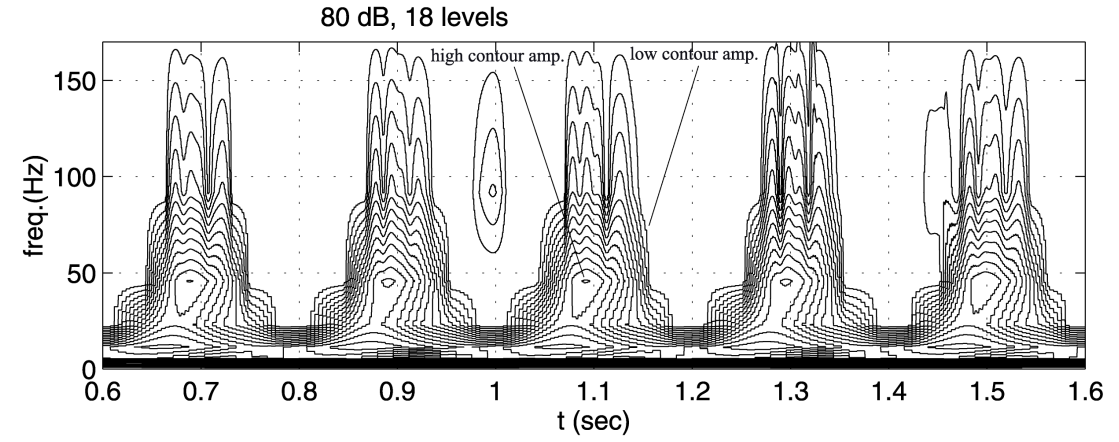

Figure 7: Wavelet analysis of the experimental stick-slip acceleration: (a) time-domain response, and (b) wavelet contour plot.

\subsection{Wavelet Contour Plots of the Experimental Stick-Slip Signal}

An experimental stick-slip acceleration signal is shown in Figure 7(a). The corresponding wavelet contour plot is presented in Figure 7(b). It can be seen that a local maximum (circular contour) similar to the one appearing in Figures 5(b) and 6(b) occurs in Figure 7(b) at about $45 \mathrm{~Hz}$. This local maximum indicates a nearly sinusoidal oscillation based on the observations made from Figures 5(b) and 6(b). The ungrouped contour structures, existing around $t=1 \mathrm{sec}$ and $t=1.45 \mathrm{sec}$, are the results of the irregularities in response. These are possibly caused by the surface roughness. Furthermore, there are several grouped spike structures occurring during each transition phase. The grouped spikes are consistent with those seen in the simulation of the compliant contact model with micro stick-slip events, namely Figure 6(b).

The $45 \mathrm{~Hz}$ transition oscillation can be used together with the mass of the experimental slider, $m=2.42 \mathrm{~kg}$, to obtain the same estimated contact stiffness as in the simulations. This stiffness approximation is limited by the resolution of the contour plots, but it is in approximate agreement with the contact stiffness obtained by examining features in friction measurements [1].

The presence of noise in the experimental signal conceals its wavelet representation; hence, quantitative comparison between Figure 6(b) and Figure 7(b) is not a trivial task. Nevertheless, the qualitative characteristics of these two wavelet contour plots definitely show some consistencies. 


\subsection{Discussion}

We have shown the signatures addressed in the wavelet transition plots of the Coulomb law, the state-variable law, and the compliant-contact model with two different degrees of damping. Each model shows the presence of high-frequency content at the onset of sticking. In the Coulomb model, this corresponds to a step change in the acceleration, after which the high-frequency contours in the wavelet transform go away. In the dynamical models the sudden high-frequency content corresponds to a sudden change to high-frequency transition oscillations, during which much high-frequency content is sustained in the wavelet contour plots. Thus, the wavelet contour plot in Figure 4(b) reflects the fact that there is no oscillation involved in the Coulomb friction case. Since the wavelet contours indicate transition oscillations in the experiment, it suggests (without surprise) that the dynamical friction models are better at accommodating this experimental feature.

If the transition oscillation is close to a damped sinusoid, there will be a local maximum corresponding to the transition-oscillation frequency. From this point of view, the transition oscillation occurring in the state-variable model case is not close to a sinusoidal function. The existence of the local maximum in the compliant-contact models and the experiment, but not in the state-variable model, provides diagnostic evidence in favor of the compliant-contact model for the physical system.

The wavelet contour plots also detect the presence of two-scale stick slip, in which case the micro stick-slip transitions each gives birth to a spike of high-frequency content in the wavelet contours. The presence of these micro-stick-slip spikes in the experiment as well as in the compliant-contact model with lighter damping, but not in the model with slightly larger damping, indicates that lighter damping is favorable in modeling the physical system.

Thus, we are able to use qualitative features in the wavelet contours to assist in the evaluation of friction models. In some cases, quantitative features are also useful. In our study, we used the local maximum in the compliant-contact models, as an indicator of the transition-oscillation frequency, together with the slider mass, to obtain realistic estimates of the contact stiffness. Higher resolution in the wavelet contours would lead to a more accurate estimation of the local maximum, and hence the contact stiffness.

\section{CONCLUSIONS}

In this investigation, the wavelet transform was used to explore time-frequency transition features of the numerical and experimental stick-slip signals. Wavelet contour features were compared between various simulated friction models and real stick-slip data. While simulations of several models can depict the transition oscillations, the compliant-contact model with light damping captures most of the characteristics of the experimental results. The wavelet transform produced signatures that distinguished the nature of the transition oscillations and the occurrence of micro stick-slip events. These features were used to evaluate the friction models.

The wavelet transform has been known to be applicable to the analysis of transient time signals. 
This work shows that the wavelet transform applied to acceleration response data can be a useful tool in the evaluation of friction models in oscillators. Elements of the evaluation can be qualitative, such as in identifying the presence of spikes and local maxima, or quantitative, as in the identification of transition oscillation frequencies.

\section{ACKNOWLEDGEMENTS}

The author Liang J.-W. is grateful of support from National Science Council of Taiwan, Republic of China (NSC90-2218-E-131-003). The authors are also grateful of support from the National Science Foundation (CMS-9624347). The authors thank Prof. Alan Haddow of MSU for discussions about wavelet transforms.

\section{REFERENCES}

[1] Liang, J.-W. and Feeny, B. F., 1998, "Dynamical friction behavior in a forced oscillator with a compliant contact," Journal of Applied Mechanics, 65(1), pp.250-257.

[2] Den Hartog, J. P., 1931, "Forced Vibration with Combined Coulomb and Viscous Damping," Transactions of the American Society of Mechanical Engineering, Vol.53, pp.107-115.

[3] Hundal, M. S., 1979, "Response of a Base Excited System with Coulomb and Viscous Friction," Journal of Sound and Vibration, Vol.64, pp.371-378.

[4] Shaw, S. W., 1986, "On the Dynamic Response of a System with Dry Friction," Journal of Sound and Vibration, Vol.108, No.2, pp.305-325.

[5] Hinrichs, N., Oestreich, M., and Popp, K., 1998, "On the modeling of friction oscillators," Journal of Sound and Vibration, 216(3), pp.435-459.

[6] Sanliturk, K. Y. and Ewins, D. J., 1996, "Modeling Two-Dimensional Friction Contact and its Application Using Harmonic Balance Method," Journal of Sound and Vibration, Vol.193, No.2, pp.511-524.

[7] Griffin, J. H., 1980, "Friction Damping of Resonant Stresses in Gas Turbine Engine Airfoils," Journal of Engineering for Power, Vol.102, pp.329-333.

[8] Ferri, A. A. and Heck, B. S., 1998 "Vibration Analysis of Dry Friction Damped Turbine Blades Using Singular Perturbation Theory," Journal of Vibration and Acoustics, Vol.120, No.2, pp.588-595.

[9] Wang, J.-H., 1996, "Design of a Friction Damper to Control Vibration of Turbine Blades," Dynamics with Friction: Modeling, Analysis, and Experiments, A. Guran, F. Pfeiffer and K. Popp eds., World Scientific, Singapore, pp.169-195.

[10] Ruina, A., 1980, "Friction Laws and Instabilities: A Quasistatic Analysis of Some Dry Frictional Behavior," Ph.D. Thesis, Division of Engineering, Brown University.

[11] Rice, J. R., and Ruina, A. L., 1983, "Stability of Steady Friction Slipping," Journal of Applied Mechanics, 50, pp.343-349.

[12] Dieterich, J. H., 1979, "Modeling of Rock Friction: 1. Experimental Results and Constitutive Equations," J. of Geophysical Research, 84(B5), pp.2161-2168.

[13] Liang, J.-W. and Feeny, B. F., 1995, "Wavelet Analysis of Stick-Slip in an Oscillator with Dry Friction," Proceedings of the ASME Conference, Friction Damping and Friction-Induced Vibration Symposium, De-Vol. 84-1, Vol.3, Part A, Boston, MA.

[14] Jalali P, Polashenski W, Tynjala T, Zamankhan P, 2002, "Particle Interactions in a Dense Monosized Granular Flow" Physica D 162 (3-4): 188-207.

[15] Knigge B, and Talke F.E., 2001, “ Slider Vibration Analysis at Contact using Time-Frequency 
Analysis and Wavelet Transforms," ASME, Journal of Tribology, 123 (3): 548-554.

[16] Basu, B. and Gupta, V. K., 2000, "Wavelet-Based Non-Stationary Response Analysis of a Friction Base-Isolated Structure," EARTHQUAKE ENGINEERING \& STRUCTURAL DYNAMICS 29 (11): 1659-1676 NOV 2000

[17] Kishimoto, K., Inoue, H., Hamada, M., and Shibuya, T., 1995, “Time Frequency Analysis of Dispersive Waves by Means of Wavelet Transform," Journal of Applied Mechanics, 62, pp.841-846.

[18] Önsay, T. and A. G. Haddow, 1994, "Wavelet Transform Analysis of Transient Wave Propagation in a Dispersive Medium," Journal of Acoustics Society of American, 95(3), pp.1441-1449.

[19] Staszewski, W. J., 1997, "Identification of Damping in MDOF Systems using Time-Scale Decomposition," Journal of Sound and Vibration, 203(2), pp.283-305.

[20] Morlet, J. G., Arens, E., Fourgeau, E., Giard, D., 1982, "Wave Propagation and Sampling Theory-Part I: Complex Signal and Scattering in Multi-layered Media," Geophysics.

[21] Mallat, S., 1998, A Wavelet Tour of Signal Processing, Academic Press, San Diego, CA.

[22] Strang, G. and Nguyen, T., 1996, Wavelets and Filter Banks, Wellesley-Cambridge Press, Wellesley, MA.

[23] Misiti, M., Misiti, Y., Oppenheim, G., and Poggi, J.-M., 1996, Wavelet Toolbox for Use with MATLAB: User's Guide, The MATHWORKS Inc.

[24] Newland, D. E., 1994, "Wavelet Analysis of Vibration Part II: Wavelet Maps," Journal of Vibration and Acoustics, 116, pp.417-425.

[25] Liang, J.-W., 1996, Characterizing the Low-Order Friction Dynamics in a Forced Oscillator, $\mathrm{PhD}$ thesis, College of Engineering, Michigan State University.

[26] Dahl, P., 1968, "A Solid Friction Model," Tech. Rep. TOR-158 (3107-18), The Aerospace Corporation, EI Segundo, CA.

[27] Feeny, B. F., and Moon, F. C., 1994, "Chaos in a Forced Dry-Friction Oscillator: Experiments and Numerical Modeling," Journal of Sound and Vibration, 170(3), pp. 303-323. 